\title{
On the Physical Conditions in AGN Optical Jets
}

\author{
Riccardo Scarpa $^{\mathrm{a}, 1}$, C. Megan Urry ${ }^{\mathrm{a}, 1}$ \\ ${ }^{a}$ Space Telescope Science Institute, 3700 San Martin Dr., Baltimore, MD 21218
}

\begin{abstract}
The energy budget of all known optical jets is discussed. It is found that to power the extended radio lobes of radio galaxies, the jet bulk motion on kpc scales must be relativistic, on average. Based on various constraints, a "most probable" region centered around $\Gamma_{b u l k} \sim 7.5$ and $\theta \sim 20^{\circ}$ is found. Because of the consequent relativistic beaming, the rest frame magnetic field is lower and electron lifetimes longer. Combining the effects of time dilation and lower emission rate, the electron diffusion length becomes fully consistent with the deprojected jet length, without the need for reacceleration.
\end{abstract}

\section{Introduction}

To emit at optical-UV frequencies in a field of $B \sim 10^{-4} \mathrm{G}$, typical of the magnetic fields found in AGN jets on kpc scales, electrons must have Lorentz factors $\gamma \gtrsim 10^{6}$ and have diffusion times of a few hundred years. Despite the shortness of the expected synchrotron cooling times, the jets are long and there is no indication of strong steepening of the radio-to-optical spectral index as the distance from the nucleus increases (Sparks et al. 1996; Scarpa et al. 1999). Therefore, either electrons are continuously reaccelerated or the magnetic field is weaker than the equipartition value, as may be the case if relativistic beaming is important.

To shed light on this issue we analyze the energy budget of all known optical jets, requiring they transport on average as much energy as needed to explain the existence of extended radio lobes. Our approach relies on the hypothesis that the extended radio structures are powered by jets (Blandford \& Königl 1979), and on the fact that, even if rare, optical jets are discovered in all kinds of radio sources (of both FRI and FRII morphologies and powers).

$\overline{1}$ E-mail: scarpa@stsci.edu; cmu@stsci.edu 
Table 1

Relevant Properties of Optical Jets ${ }^{(a)}$

\begin{tabular}{|c|c|c|c|c|c|c|c|c|c|c|c|c|c|}
\hline Name & $\begin{array}{l}\mathrm{Cl} . \\
\text { (b) }\end{array}$ & $\mathrm{z}$ & $\begin{array}{l}\nu_{R} \\
\text { (c) }\end{array}$ & $\begin{array}{r}F_{R} \\
\text { (c) }\end{array}$ & $\begin{array}{l}\nu_{O} \\
\text { (d) }\end{array}$ & $\begin{array}{l}F_{O} \\
\text { (d) }\end{array}$ & $\begin{array}{r}1 \\
\text { (e) }\end{array}$ & $\begin{array}{l}\text { Vol } \\
\text { (f) }\end{array}$ & $\begin{array}{r}\alpha_{R O} \\
\quad(\mathrm{~g})\end{array}$ & $\begin{array}{l}\text { ref. } \\
\text { (h) }\end{array}$ & $\begin{array}{r}J \\
\text { (i) }\end{array}$ & $\begin{array}{r}\mathrm{B}_{o} \\
(\mathrm{j})\end{array}$ & $\begin{array}{l}\mathrm{B}_{r} \\
(\mathrm{k})\end{array}$ \\
\hline M87 & I & 0.004 & 15 & 4.2 & 6.88 & 1960 & 2.1 & 62.7 & -0.71 & 2,24 & $>450$ & 31 & 12 \\
\hline $3 \mathrm{C} 15$ & $\mathrm{I} / \mathrm{II}$ & 0.073 & 2.78 & 0.21 & 5.50 & 5.9 & 3.2 & 65.4 & -0.86 & 21 & $>50$ & 8.7 & 3.3 \\
\hline $3 \mathrm{C} 66 \mathrm{~B}$ & $\mathrm{I} / \mathrm{II}$ & 0.021 & 15 & 0.04 & 8.17 & 6.5 & 3.0 & 65.1 & -0.80 & $2,4,6,25$ & $\gtrsim 40$ & 4.5 & 1.7 \\
\hline $3 \mathrm{C} 78$ & I & 0.029 & 1.66 & 0.8 & 4.34 & 33 & 1.1 & 62.2 & -0.81 & 12,26 & $>40$ & 53 & 20 \\
\hline $3 \mathrm{C} 120$ & I & 0.033 & 5.0 & 0.05 & 6.88 & 14 & 0.32 & 64.6 & -0.69 & $11,13,27$ & $>1000$ & 6.3 & 2.4 \\
\hline $3 \mathrm{C} 200$ & II & 0.458 & 5.0 & 0.076 & 4.16 & 18 & 0.32 & 65.9 & -0.74 & 9,10 & $>37.5$ & 15 & 5.8 \\
\hline $3 \mathrm{C} 212$ & II & 1.049 & 8.33 & 0.04 & 9.09 & 0.19 & 0.85 & 67.1 & -1.06 & 7 & $\ldots$ & 56 & 21 \\
\hline $3 \mathrm{C} 245$ & II & 1.029 & 5.0 & 0.045 & 9.09 & 0.17 & 31.7 & 67.2 & -1.03 & $7,8,22$ & $\ldots$ & 84 & 32 \\
\hline $3 \mathrm{C} 264$ & I & 0.020 & 15 & 0.2 & 4.16 & 33 & 0.32 & 62.1 & -0.85 & 1,2 & $>37$ & 89 & 34 \\
\hline $3 \mathrm{C} 273$ & II & 0.158 & 15 & 2.41 & 5.50 & 57 & 4.8 & 64.8 & -1.07 & $2,5,18$ & $>5300$ & 5.8 & 2.2 \\
\hline $3 \mathrm{C} 346^{(l)}$ & II & 0.161 & 15 & 0.08 & 4.16 & 11 & 11.0 & 62.8 & -0.87 & 14,15 & $\ldots$ & 5.6 & 2.1 \\
\hline $3 \mathrm{C} 371$ & II & 0.051 & 1.6 & 0.18 & 5.50 & 15 & 28.9 & 65.9 & -0.74 & $17,19,23$ & $>700$ & 17 & 6.4 \\
\hline $0521-365$ & I & 0.055 & 15 & 0.1 & 4.16 & 60 & 21.9 & 65.9 & -0.73 & $3,16,23$ & $>10$ & 14 & 5.1 \\
\hline $2201+044$ & I & 0.027 & 5.0 & 0.009 & 4.16 & 0.57 & 2.05 & 63.9 & -0.85 & 20,23 & $\ldots$ & 7.9 & 3.0 \\
\hline
\end{tabular}

(a) All values are in the observer frame. (b) Fanaroff \& Riley morphology class. (c) Radio frequency in $\mathrm{GHz}$ and radio flux in Jy. (d) Optical frequency in $10^{14} \mathrm{~Hz}$ and optical flux in $\mu \mathrm{Jy}$. (e) Projected jet length in kpc. (f) Logarithm of jet volume in $\mathrm{cm}^{3}$, estimated assuming cylindrical symmetry. (g) Radio to optical spectral index assuming $F_{\nu} \propto \nu^{\alpha}$.

(h) References for fluxes, size, and $J: 1$ ) Baum et al. 1997; 2) Crane et al. 1993; 3) Keel 1986; 4) Fraix-Burnet et al. 1989; 5) Bahcall et al. 1995; 6) Macchetto et al. 1991; 7) Ridgway \& Stockton 1997; 8) Laing 1989; 9) Burns et al. 1984; 10) Bogers et al. 1994; 11) Hjorth et al. 1995; 12) Sparks et al. 1995; 13) Fraix-Burnet et al. 1991; 14) van Breugel et al. 1992; 15) Dey \& van Breugel 1994; 16) Macchetto et al. 1991; 17) Wrobel \& Lind 1990; 18) Davis et al. 1991; 19) Akujor et al. 1994; 20) Laurent-Muehleisen et al. 1993; 21) Leahy et al. 1997; 22) Saikia et al. 1990; 23) Scarpa et al. 1999.; 24) Stiavelli et al. 1992; 25) Fraix-Burnet 1997; 26) Saikia et al. 1986; 27) Walker et al. 1987.

(i) Jet/counter-jet luminosity ratio as published or derived by us from published radio maps. (j) "Observed" equipartition magnetic field in units of $10^{-5} \mathrm{G}$, computed without transforming to the rest frame and without beaming. (k) "Rest frame" equipartition magnetic field in units of $10^{-5} \mathrm{G}$, computed assuming $\Gamma=7.5$ and $\theta=20^{\circ}$ for all jets. (1) Only knot "c" as defined in van Breugel et al. (1992) considered.

\section{Theoretical Considerations and Available Data}

Standard formulae for synchrotron emission are used (Pacholczyk 1970). The synchrotron emission is assumed to extend, in the observer frame, from $\nu_{1}=$ $10^{7}$ to $\nu_{2}=10^{15} \mathrm{~Hz}$, following a single power law $\left(F_{\nu} \propto \nu^{\alpha}\right)$ of constant spectral index $\alpha$. The electron distribution in energy space is $N(E)=N_{0} E^{p}$, where $p=2 \alpha-1$. Each electron emits in a narrow range of frequencies centered on $\nu=c_{1} B E^{2}$, where $c_{1}=\frac{3 e}{4 \pi m^{3} c^{5}}$, at a rate of $d E / d t=c_{2} B E^{2}$, where $c_{2}=\frac{2 e^{4}}{3 m^{4} c^{7}}$. In the presence of relativistic beaming, rest frame $\left(L_{R}\right)$ and observed $(L)$ jet luminosities are related by $L=L_{R} \delta^{3}$ (as appropriate for a continuous jet; it would be $L=L_{R} \delta^{4}$ for a moving sphere), where $\delta=[\Gamma(1-\beta \cos \theta)]^{-1}$ is the Doppler beaming factor. The rest-frame luminosity is $L_{R}=\int_{E_{1}}^{E_{2}} \frac{d E}{d t} N(E) d E$, where $E_{1}$ and $E_{2}$ are the cutoffs of the electron energy distribution corresponding to $\nu_{1}$ and $\nu_{2}$. After integrating, $L_{R}$ depends on $B$ and $N_{0}$, so that a second equation is necessary to solve completely for jet properties. This can be obtained by imposing equipartition of energy, in which case we have $\frac{B^{2} \phi V}{8 \pi}=(1+k) E_{e}$, where $\phi$ is the magnetic field filling factor, $\mathrm{V}$ is the source volume, and $E_{e}$ is the total electron energy. The proton energy, which remains unconstrained, is accounted for by the proton-to-electron energy ratio $k$. For consistency with previous works, we set $k=0$ (assigning no energy 
to the protons). Solving for $B$ and $N_{0}$ gives the rest-frame number density $n=\frac{L}{c_{2} B^{2}<E^{2}>V \delta^{3}}$ of the emitting electrons, to be used to derive the kinetic energy transported in the jet, which is $L_{k}=\pi R^{2} \Gamma^{2} \beta c n<E>(1+k)$ (Celotti \& Fabian 1993). Here $\Gamma$ is the Lorentz factor of the bulk motion, $\beta c$ the plasma speed, and $R$ the jet radius. As before, the term $(1+k)$ accounts for the proton energy. Under these quite standard assumptions, the flux at 2 frequencies and the bulk speed of the plasma suffice to evaluate the jet kinetic energy. Relevant data for all known optical jets are summarized in Table 1. Interestingly, optical jets are discovered in radio sources of all types (Column 2), and have remarkably similar radio-to-optical spectral indexes $\left(\alpha_{R O} \sim-0.8\right)$, consistent with radio observations where $90 \%$ of the jets have $-0.5<\alpha<-0.9$ (Bridle \& Perley 1984). This indicates a high degree of homogeneity of all jets, so that it is reasonable to compare their energy budgets with those for radio lobes observed in a large sample of radio sources.

\section{Bulk Jet Speed}

Relativistic beaming elegantly explains both jet one-sidedness and superluminal motion, observed routinely on parsec scales (Zensus \& Pearson 1987; Readhead 1993), and also on kpc scales in the jet of M87 (Biretta et al. 1999). Assuming the two sides of the jet are intrinsically identical (Rees 1978; Shklovsky 1970; Saslaw \& Whittle 1988; Laing 1988), the Lorentz factor of the bulk speed can then be derived (Scheuer \& Readhead 1979) from the jet/counterjet luminosity ratio $J=\left(\frac{1+\beta \cos \theta}{1-\beta \cos \theta}\right)^{(2-\alpha)}$, where $\theta$ is the angle between jet speed and line of sight, and the exponent $(2-\alpha)$ is appropriate for a continuum jet (it would be $3-\alpha$ for discrete emitting blobs; see Lind \& Blandford 1985).

The dependence of $J$ on jet inclination $\theta$ is shown in Figure 1. It is seen that $J$ easily reaches very large values even for modest bulk speed, but for large inclinations it remains finite and quite small even for $\Gamma \rightarrow \infty$, so that $J$ can be effectively used to constrain $\theta$. From Table 1 the median lower limit of $J$ for optical jets is 40 , implying median inclinations $\lesssim 55^{\circ}$ and $\Gamma>1.1$.

\section{Jet Versus Lobe Kinetic Energy}

The kinetic luminosity depends on the density and average energy of the emitting electrons, as well as the bulk speed of the plasma. These quantities are therefore constrained if the kinetic luminosity can be estimated in an independent way. Here we require jets to transport enough energy for powering an average radio lobe. Setting all relevant parameters as before (i.e., same low 

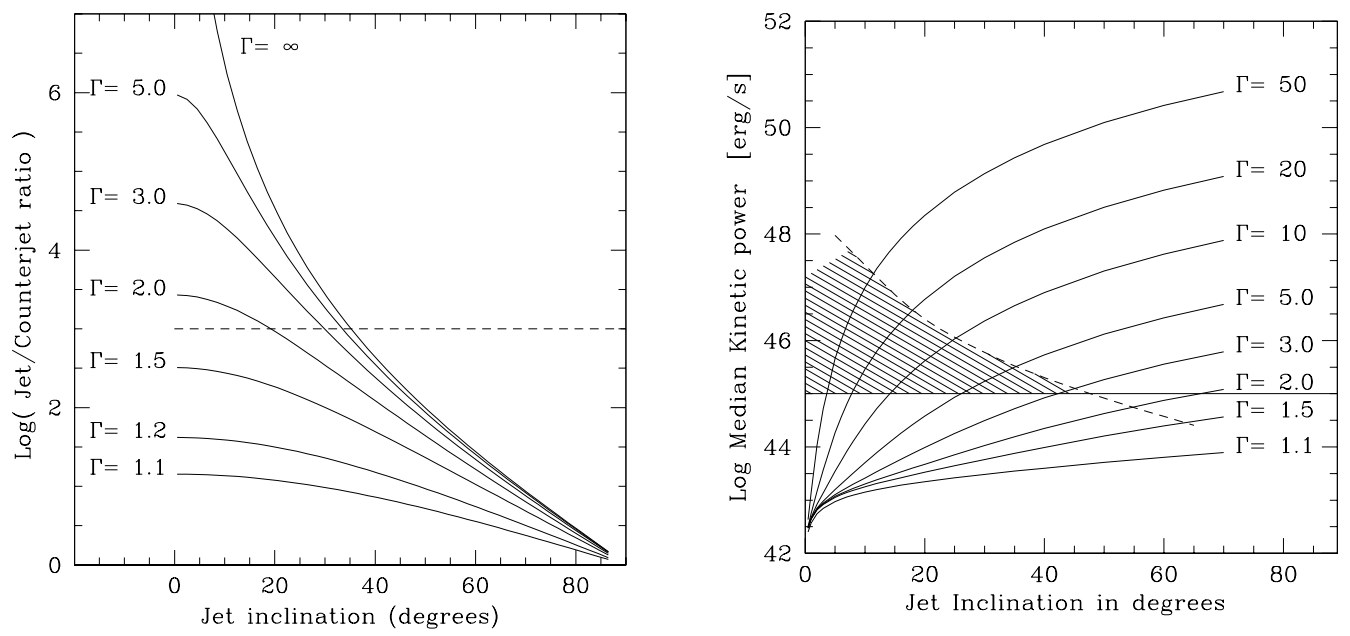

Fig. 1. Left: Jet/counterjet luminosity ratio $J$ for an intrinsically symmetric jet, as function of jet inclination, for different Lorentz factors for the bulk motion. Observed lower limits to $J$ give upper limits on $\theta$ and/or lower limits on $\Gamma$. For example the dashed line correspond to the available lower limits on $J$ for 3C 120 . This implies a jet inclination $<35^{\circ}$ and $\Gamma>1.7$. Right: Median kinetic power in equipartition for the 14 optical jets versus jet inclination $\theta$ and for several values of $\Gamma$. The horizontal line at $L_{k i n}=10^{45} \mathrm{erg} / \mathrm{s}$ indicates the median power estimated for radio lobes (Rawlings \& Saunders 1991). The dashed line is the locus of beaming $\delta=1$, separating beamed (below) from de-beamed (above) jets. Combining these two constraints, allowed $\theta$ and $\Gamma$ are restricted to the shaded area.

frequency cutoff, $k=0$, and $\phi=1$ ), a median kinetic power of $<L_{k i n}>=$ $10^{45} \mathrm{erg} / \mathrm{s}$ was found for a large sample of radio galaxies including both weak and powerful radio sources (Rawlings \& Saunders 1991). The high frequency cutoff is much higher for optical jets than radio lobes, but this should have no effect on the energy budget because for $p<-2(\alpha<-0.5)$ the energetics are dominated by the low energy particles. Comparing the median kinetic power of all optical jets with that of radio lobes (Figure 1), it is found that for very low bulk speeds $(\Gamma \lesssim 2)$, the median kinetic energy for this sample of jets is at least one order of magnitude smaller than needed to power average lobes, independently of inclination angle. Very small inclinations are also excluded, because for small $\theta$ the beaming is so strong that the rest frame luminosity of the jet is very small, and the kinetic energy significantly reduced.

The maximum speed of the plasma can be loosely constrained if that jets with enhanced rather than dimmed emission are preferentially discovered. For any given value of $\theta$, as soon as $\Gamma$ becomes larger than the value defined by $\Gamma-\sqrt{\Gamma^{2}-1} \cos \theta=1$, the emission is de-amplified, severely reducing our probability of discovering the jet. In this way we derive a "most probable" region, centered on $\theta \sim 20^{\circ}$ and $\Gamma \sim 7.5$, where the average kinetic energy carried by (optical) jets is fully consistent with the requirement imposed by radio lobes. The basic conclusion is that jets should to be relativistic at kpc 

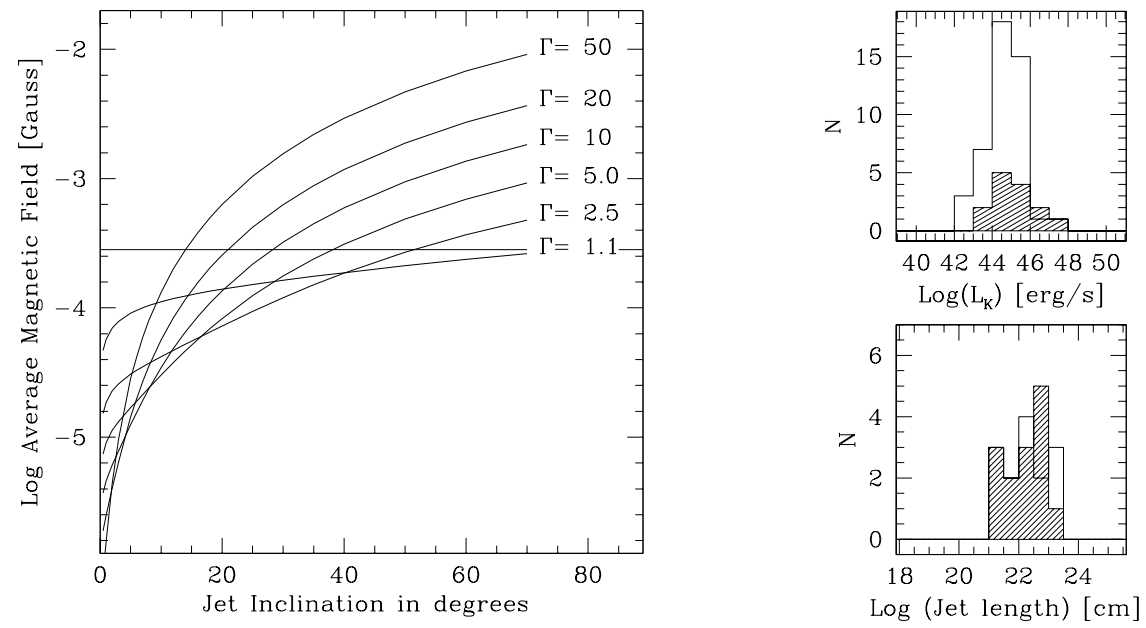

Fig. 2. Left: Average equipartition magnetic field for the whole sample versus jet inclination for several values of $\Gamma$. The horizontal line at $B=2.8 \times 10^{-4} \mathrm{G}$ indicates the median magnetic field derived directly from values given in Table 1 without including beaming. Upper right: Kinetic energy distribution of jets (dashed histogram) compared to radio lobes power (open histogram), evaluated assuming equipartition and $\theta=20^{\circ}$ and $\Gamma=7.5$ for all jets. The median jet kinetic energy is $10^{45} \mathrm{erg} / \mathrm{s}$ as for radio lobes (Rawlings \& Saunders 1991). Lower Right: Distribution of estimated electron diffusion length $c t_{1 / 2} \Gamma$ for electrons emitting at $10^{15}$ $\mathrm{Hz}$ in the observer frame (dashed histogram), compared with the observed deprojected jet lengths (open histogram). The diffusion length was computed assuming equipartition the same values of $\Gamma$ and $\theta$ as before.

scales.

\section{Conclusions}

Comparing the kinetic power (as derived assuming equipartition) for all known optical jets with that for typical radio lobes suggests the presence of relativistic bulk motion of the emitting plasma at kiloparsec scales. In a non-relativistic scenario the estimated kinetic jet luminosity is at least one order of magnitude less than needed to power the lobes.

This has important consequences. Indeed, at face value, the data in Table 1 imply strong magnetic fields and short electron lifetimes, leading to the conclusion that electron reacceleration is necessary to explain the optical emission on kpc scales (e.g., Meisenheimer et al. 1996). The analysis here points to an opposite possibility. Indeed, the constraints on the kinetic luminosity allow $\Gamma$ and $\theta$ to lie within a "most probable" region (Fig. 1), centered near $\Gamma \sim 7.5$, corresponding to a highly relativistic bulk speed. Because of relativistic beaming, the rest-frame magnetic field is reduced (Table 1), and the 
electron lifetimes are lengthened because of the lower energy losses and time dilation. In these conditions the de-projected length $l / \sin \theta$ of the jets is fully consistent with the electron diffusion length $c t_{1 / 2} \Gamma$, without the need for reacceleration (Figure 2), explaining the very nearly uniform $\alpha_{R O}$ observed in the jets of M87 (Sparks et al. 1996) and PKS 0521-365 (Scarpa et al. 1999).

It is a pleasure to thanks G. Ghisellini, L. Maraschi, and F. Macchetto for helpful and encouraging comments. Support for this work was provided by NASA through grant GO-06363.01-95A from the Space Telescope Science Institute, which is operated by AURA, Inc., under NASA contract NAS 5-26555.

\section{References}

Akujor C.E., Lüdke E., Browne I.W.A., et al. 1994 A\&AS 105, 247

Bachall J.N., Kirhakos S., Schneider D.P., et al. 1995, ApJ 452, L91

Baum S.A., O’Dea C.P., Giovannini G. et al. 1997, ApJ 483, 178

Biretta J.A., Perlman E., Sparks W.B. \& Macchetto F. 1999, AAS meeting N. 193, 07.09

Blandford R.D. \& Königl A. 1979 ApJ 332, 34

Bogers W.J., Hes R., Barthel P.D. \& Zensus J.A. 1994, A\&AS 105, 91

Bridle A.H. \& Perley R.A. 1984, ARA\&A, 22, 319

Burns J.O., Basart J.P., De Young D.S. \& Ghiglia D.C. 1984, ApJ 283, 515

Celotti A. \& Fabian A.C. 1993, MNRAS 264, 228

Crane P., Peletier R., Baxter D. et al. 1993, ApJ 402, L37

Davis R.J., Unwin S.C., Muxlow T.W.B. 1991, Nature 354, 374

Dey A. \& van Breugel 1994, AJ 107, 1977

Fraix-Burnet D. 1997, MNRAS 284, 911

Fraix-Burnet D., Nieto J.L., Leliévre G., et al. 1989 ApJ 336, 121

Fraix-Burnet D., Golombek D. \& Macchetto D. 1991, AJ 102, 562

Hjorth J., Vestergaard M., So rensen A.N. \& Grundahl F. 1995, ApJ 452, L17

Keel W.C. 1986 ApJ 302, 296

Laing R.A. 1988, Nature 331, 149

Laing R. 1989, in "Hot spots in extragalactic radio sources", Springer-Verlag, p. 27 
Laurent-Muehleisen S.A., Kollgaard R.I., Moellenbrock G.A. \& Feigelson E.D. 1993, AJ 106, 875

Leahy J.P., Black A.R.S. \& Dennet-Thorpe J. 1997, MNRAS 291, 20L

Lind, K.R. \& Blandford R.D. 1985, ApJ 295, 358

Macchetto F., Albrecht R., Barbieri C., et al. 1991 ApJ 369, L55

Martel A.R., Sparks W.B., Macchetto F., et al. 1998 ApJ 496, 203

Meisenheimer K., Röser H.-J. \& Schlötelburg M. 1996, A\&A 307, 61

Nilsson K., Heidt J., Pursimo T. et al. 1997 ApJ 484, L107

Pacholcsyk A.G. 1970, "Radio Astrophysics", printed by W.H. Freeman and Company, San Francisco.

Rawlings S \& Saunders R. 1991, Nature 349, 138

Readhead A.C.S. 1993, in Sub-Arcsecond Radio Astronomy, Cambridge: Cambridge

Univ. Press, pp. 173

Rees M. J 1978 MNRAS 184, 61

Ridgway S.E. \& Stockton A. 1997, AJ 114, 511

Saikia D.J., Subrahmanya C.R., Patnaik A.R. et al. 1986, MNRAS 219, 545

Saikia D.J., Junor W., Cornwell T.J. et al. 1990, MNRAS 245, 408

Saslaw W.C. \& Whittle M. 1988, ApJ 325, 142

Scarpa R., Urry C.M., Falomo R. \& Treves A. 1999, ApJ 526, in press

Scarrot S.M. \& Warren-Smith R.F. 1987, MNRAS 228, 35p

Scheuer P.A.G. \& Readhead A.C.S. 1979, Nature 277, 182

Scheuer P.A.G. 1974, MNRAS 166, 513

Shklovsky J. 1970, Nature 228, 1174

Sparks W.B., Biretta J.A. \& Macchetto F. 1996, ApJ 473, 254

Sparks W.B., Golombek D., Baum S.A. et al. 1995, ApJ 450, L55

Stiavelli M., Biretta J., Møller P. \& Zeilinger W.W. 1992 Nature 355, 802

van Breugel W.J.M., Fanti C., Fanti R., et al. 1992, A\&A 256, 56

Walker R.C., Bensons J.M. \& Unwin S.C. 1978, in Superluminal Radio Sources, ed. J.A. Zensus \& T.J. Pearson, pg. 48 ApJ 278, 521

Wrobel J.M. \& Lind K.R. 1990, ApJ 348, 135

Zensus J.A. \& Pearson T.J. 1987, in Superluminal Radio Sources, Cambridge Univ. Press, pp. 1 